TITLE:

\title{
n- and p-type dopants for cubic silicon nitride
}

AUTHOR(S):

Oba, F; Tatsumi, K; Adachi, H; Tanaka, I

CITATION:

Oba, F ...[et al]. n-and p-type dopants for cubic silicon nitride. APPLIED PHYSICS LETTERS 2001, 78(11): 1577-1579

\section{ISSUE DATE:}

2001-03-12

URL:

http://hdl.handle.net/2433/39641

\section{RIGHT:}

Copyright 2001 American Institute of Physics. This article may be downloaded for personal use only. Any other use requires prior permission of the author and the American Institute of Physics. 


\title{
$\boldsymbol{n}$ - and $\boldsymbol{p}$-type dopants for cubic silicon nitride
}

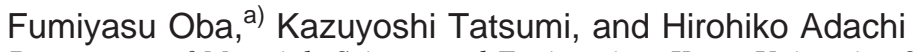 \\ Department of Materials Science and Engineering, Kyoto University, Sakyo, Kyoto 606-8501, Japan \\ Isao Tanaka \\ Department of Energy Science and Technology, Kyoto University, Sakyo, Kyoto 606-8501, Japan
}

(Received 10 November 2000; accepted for publication 13 January 2001)

\begin{abstract}
The formation and ionization energies of impurities in cubic silicon nitride are investigated through first-principles calculations. Among the elements in the groups III to VI, P and O are preferable for $n$-type doping, while $\mathrm{Al}$ is favorable for $p$-type doping in terms of the formation and ionization energies. The compensation of doped carriers associated with the incorporation of these impurities into anti and interstitial sites can be suppressed if appropriate growth conditions are chosen. (C) 2001 American Institute of Physics. [DOI: 10.1063/1.1354667]
\end{abstract}

Silicon nitride $\left(\mathrm{Si}_{3} \mathrm{~N}_{4}\right)$ is important as a structural component. It is also used as an insulating layer in the silicon technology. Hexagonal $\mathrm{Si}_{3} \mathrm{~N}_{4}$ crystals $(\alpha$ and $\beta$ ) as well as amorphous films have thus far been utilized for these applications. In 1999, a third crystalline phase of $\mathrm{Si}_{3} \mathrm{~N}_{4}$, i.e., $\mathrm{Si}_{3} \mathrm{~N}_{4}$ in the cubic spinel structure $\left(c-\mathrm{Si}_{3} \mathrm{~N}_{4}\right)$, was discovered. ${ }^{1} \mathrm{~A}$ first-principles calculation within the local density approximation then suggested that $c-\mathrm{Si}_{3} \mathrm{~N}_{4}$ has a direct band gap of $3.45 \mathrm{eV}^{2}$ This is in contrast to wider and indirect band gaps of $\alpha$ - and $\beta-\mathrm{Si}_{3} \mathrm{~N}_{4}$ estimated through similar calculations. ${ }^{3}$ The direct band gap implies the potential applications of $c-\mathrm{Si}_{3} \mathrm{~N}_{4}$ as optical or electronic devices in addition to the structural applications. In order to use $c-\mathrm{Si}_{3} \mathrm{~N}_{4}$ as a semiconductor, it is essential to find good dopants. In the present letter, we report the results of first-principles calculations on various kinds of $n$ - and $p$-type dopants for $c-\mathrm{Si}_{3} \mathrm{~N}_{4}$; the information should be useful for the search of conducting $\mathrm{Si}_{3} \mathrm{~N}_{4}$ that may rival the preexisting semiconductors.

The calculations were performed for 56-atom supercells containing impurities by a first-principles plane-wave pseudopotential method ${ }^{4}$ within the generalized gradient approximation. ${ }^{5}$ Ultrasoft pseudopotentials ${ }^{6}$ were employed with a plane-wave cutoff energy of $360 \mathrm{eV}$, which was confirmed to achieve the convergence of the formation energies of the impurities within $0.05 \mathrm{eV}$ for cutoff energies up to 700 $\mathrm{eV}$. Numerical integration over the Brillouin zone was carried out at the $\Gamma$ point. The positions of atoms within the second nearest neighbors of the impurities were relaxed under a constant volume condition, using the lattice constant optimized for the perfect crystal of $c-\mathrm{Si}_{3} \mathrm{~N}_{4}{ }^{7}$ The relaxation procedures were truncated when all the residual forces for the relaxed atoms were less than $0.1 \mathrm{eV} / \AA$.

As for impurity species, we consider $\mathrm{B}, \mathrm{Al}$, and $\mathrm{Ga}$ from the group III, and $\mathrm{P}$ and As from the group V to substitute for $\mathrm{Si}$. C from the group IV, and O and $\mathrm{S}$ from the group VI are adopted to substitute for $\mathrm{N}$. The other elements in these groups are not investigated since they are unlikely to be doped effectively due to the significant size mismatch. For

\footnotetext{
${ }^{a)}$ Present address: Engineering Research Institute, School of Engineering, The University of Tokyo, Bunkyo, Tokyo 113-8656, Japan; electronic mail: oba@ sigma.t.u-tokyo.ac.jp
}

the Si-site substitution, the two types of $\mathrm{Si}$ sites in the spinel structure, i.e., the tetrahedral and octahedral sites of the $\mathrm{N}$ sublattice in the face-centered cubic structure, are considered.

Using the total energies of the supercells, the formation and ionization energies of the impurities were calculated. The formation energy is given as a function of the atomic chemical potentials and the Fermi energy, i.e., ${ }^{8,9}$

$$
E_{\text {formation }}(q)=E_{T}(q)-n_{\mathrm{Si}} \mu_{\mathrm{Si}}-n_{\mathrm{N}} \mu_{\mathrm{N}}-\mu_{i}+q E_{F},
$$

where $E_{T}$ is the total energy of a supercell containing an impurity atom in a charge state $q . n_{\mathrm{Si}}$ and $n_{\mathrm{N}}$ are the number of $\mathrm{Si}$ and $\mathrm{N}$ atoms in the supercell. $\mu_{\mathrm{Si}}, \mu_{\mathrm{N}}$ and $\mu_{i}$ are the chemical potentials of $\mathrm{Si}, \mathrm{N}$ and impurity atoms. $E_{F}$ is the Fermi energy or the electron chemical potential. $\mu_{\mathrm{Si}}$ and $\mu_{\mathrm{N}}$ are variables correlated as

$$
3 \mu_{\mathrm{Si}}+4 \mu_{\mathrm{N}}=\mu_{c-\mathrm{Si}_{3} \mathrm{~N}_{4}(\text { bulk })},
$$

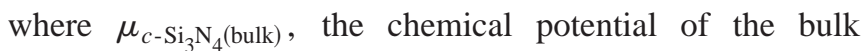
$c-\mathrm{Si}_{3} \mathrm{~N}_{4}$, is a constant value calculated as the total energy of $c-\mathrm{Si}_{3} \mathrm{~N}_{4}$ per unit formula. The total energies per atom for the bulk Si and $\alpha-\mathrm{N}_{2}$ were chosen as the upper limits of $\mu_{\mathrm{Si}}$ and $\mu_{\mathrm{N}}$, respectively. When $\mu_{\mathrm{Si}}$ and $\mu_{\mathrm{N}}$ violate these limits, the bulk $c-\mathrm{Si}_{3} \mathrm{~N}_{4}$ is thermodynamically unstable. Likewise, $\mu_{i}$ should vary within the range that the impurity element does not segregate, i.e., the upper limit is determined by the solubility limit. Assuming values of $\mu_{\mathrm{Si}}, \mu_{\mathrm{N}}$, and $\mu_{i}$ in these ranges, we can discuss the formation energies of the impurities in $c-\mathrm{Si}_{3} \mathrm{~N}_{4}$ under a growth condition.

The ionization energies were evaluated as the difference between the formation energies of the neutral and charged impurities when the Fermi energy is the same as the minimum of the conduction band ${ }^{10}$ and the maximum of the valence band for positive ionization ( $n$-type doping) and negative ionization ( $p$-type doping), respectively.

The calculated ionization and formation energies are listed in Table I. The ionization energies are those of single ionization; further ionization was found to be much higher in energy and hence unlikely for all the impurities considered here. The formation energies are calculated for the neutral charge state at the solubility limits under the two extreme conditions, i.e., the Si-rich limit $\left(\mu_{\mathrm{Si}}=\mu_{\mathrm{Si} \text { (bulk })}\right.$ and $\mu_{\mathrm{N}}$ 
TABLE I. Ionization and formation energies of impurities in $c-\mathrm{Si}_{3} \mathrm{~N}_{4}$. The formation energies are calculated for the neutral charge state at the solubility limits that are determined by the equilibrium conditions with the simple substances or the compounds shown as segregants; the energies in the parentheses are determined by the simple substances, whereas the others are determined by the compounds.

\begin{tabular}{|c|c|c|c|c|c|c|}
\hline \multirow{2}{*}{$\begin{array}{c}\text { Doping } \\
\text { type }\end{array}$} & \multirow{2}{*}{$\begin{array}{r}\text { Impurity } \\
\text { element }\end{array}$} & \multirow{2}{*}{$\begin{array}{l}\text { Substitution } \\
\text { site }\end{array}$} & \multirow{2}{*}{$\begin{array}{l}\text { Ionization } \\
\text { energy }(\mathrm{eV})\end{array}$} & \multicolumn{3}{|c|}{ Formation energy $(\mathrm{eV})$} \\
\hline & & & & Si-rich & N-rich & Segregants \\
\hline \multirow[t]{6}{*}{$n$} & $\mathrm{P}$ & $\mathrm{Si}$ (tet) & 0.1 & 6.7 & $(0.3)$ & $\mathrm{SiP}$ \\
\hline & & $\mathrm{Si}$ (oct) & 0.4 & 6.4 & $(0.0)$ & \\
\hline & As & Si(tet) & 0.0 & 5.8 & (2.4) & SiAs \\
\hline & & $\mathrm{Si}$ (oct) & 0.8 & 5.2 & (1.8) & \\
\hline & $\mathrm{O}$ & $\mathrm{N}$ & 0.1 & 2.3 & 3.1 & $\mathrm{SiO}_{2}(\alpha$ quartz $)$ \\
\hline & $\mathrm{S}$ & $\mathrm{N}$ & 0.1 & 5.4 & (6.7) & $\mathrm{SiS}_{2}$ \\
\hline \multirow[t]{7}{*}{$p$} & B & $\mathrm{Si}($ tet $)$ & -0.1 & 4.2 & 3.4 & $c-\mathrm{BN}$ \\
\hline & & $\mathrm{Si}($ oct $)$ & 0.0 & 5.7 & 4.9 & \\
\hline & $\mathrm{Al}$ & $\mathrm{Si}$ (tet) & 0.0 & 2.6 & 1.8 & $w$-AlN \\
\hline & & $\mathrm{Si}$ (oct) & 0.1 & 2.5 & 1.7 & \\
\hline & $\mathrm{Ga}$ & Si(tet) & 0.2 & (3.7) & 2.0 & $w-\mathrm{GaN}$ \\
\hline & & $\mathrm{Si}$ (oct) & 0.5 & (4.6) & 2.9 & \\
\hline & C & $\mathrm{N}$ & 0.7 & 3.3 & (5.0) & $\beta-\mathrm{SiC}$ \\
\hline
\end{tabular}

$\left.=1 / 4 \mu_{c-\mathrm{Si}_{3} \mathrm{~N}_{4}(\text { bulk })}-3 / 4 \mu_{\mathrm{Si} \text { (bulk) }}\right)$ and the N-rich limit $\left(\mu_{\mathrm{Si}}\right.$ $=1 / 3 \mu_{c-\mathrm{Si}_{3} \mathrm{~N}_{4} \text { (bulk) }}-4 / 3 \mu_{\mathrm{N}(\text { bulk })}$ and $\left.\mu_{\mathrm{N}}=\mu_{\mathrm{N}(\text { bulk })}\right)$. The solubility limits are assumed to be the conditions that either the simple substances or the compounds shown in Table I just start to segregate. In other words, $c-\mathrm{Si}_{3} \mathrm{~N}_{4}$ doped with the impurities is in equilibrium with the simple substances or the compounds at the limits. ${ }^{11}$ For the Si-site substitution, N-rich conditions are preferable in formation energy, whereas $\mathrm{Si}$ rich conditions are preferable for the $\mathrm{N}$-site substitution, as recognized for the $\mathrm{Si}$ - and $\mathrm{N}$-rich limits in Table I. Note that the ionization energies and the differences between the formation energies for the $\mathrm{Si}$ (tet) and $\mathrm{Si}$ (oct) sites are independent of the atomic chemical potentials.

For the $n$-type doping, all the impurities exhibit the ionization energies of lower than $0.1 \mathrm{eV}$. Regarding the $\mathrm{P}$ and As impurities substituting for $\mathrm{Si}$, only the doping into the tetrahedral sites should be effective since the ionization energies for the octahedral sites are too high. When the tetrahedral sites are occupied, the formation energies at the solubility limits under the extreme N-rich condition are 0.3 and $2.4 \mathrm{eV}$ for the $\mathrm{P}$ and As impurities. The significant difference in formation energy should suggest that the $\mathrm{P}$ impurities are preferable to substitute for $\mathrm{Si}$ although the ionization energy is $0.1 \mathrm{eV}$ higher than that of the As impurities.

Concerning the N-site substitution, the $\mathrm{O}$ and $\mathrm{S}$ impurities show approximately the same ionization energies. As for the formation energies, the $\mathrm{S}$ impurities are too high even at the Si-rich limit. This implies that the $\mathrm{S}$ impurities are hard to be doped. We therefore suggest that the $\mathrm{O}$ impurities are preferable to substitute for $\mathrm{N}$.

Turning to the $p$-type doping, the $\mathrm{B}$ and $\mathrm{Al}$ impurities at the $\mathrm{Si}$ sites show the ionization energies of lower than 0.1 $\mathrm{eV}$. In terms of the formation energies, however, the $\mathrm{Al}$ impurities are clearly preferable. The Al impurities are likely to be doped into both of the $\mathrm{Si}$ (tet) and $\mathrm{Si}$ (oct) sites in nearly the same magnitude of concentration since the formation energies for these sites are comparable. On the other hand, the $\mathrm{Ga}$ and $\mathrm{C}$ impurities exhibit high ionization and formation energies. These impurities cannot act as effective acceptors. Downloaded 30 May 2007 to 130.54.110.22. Redistribution subject

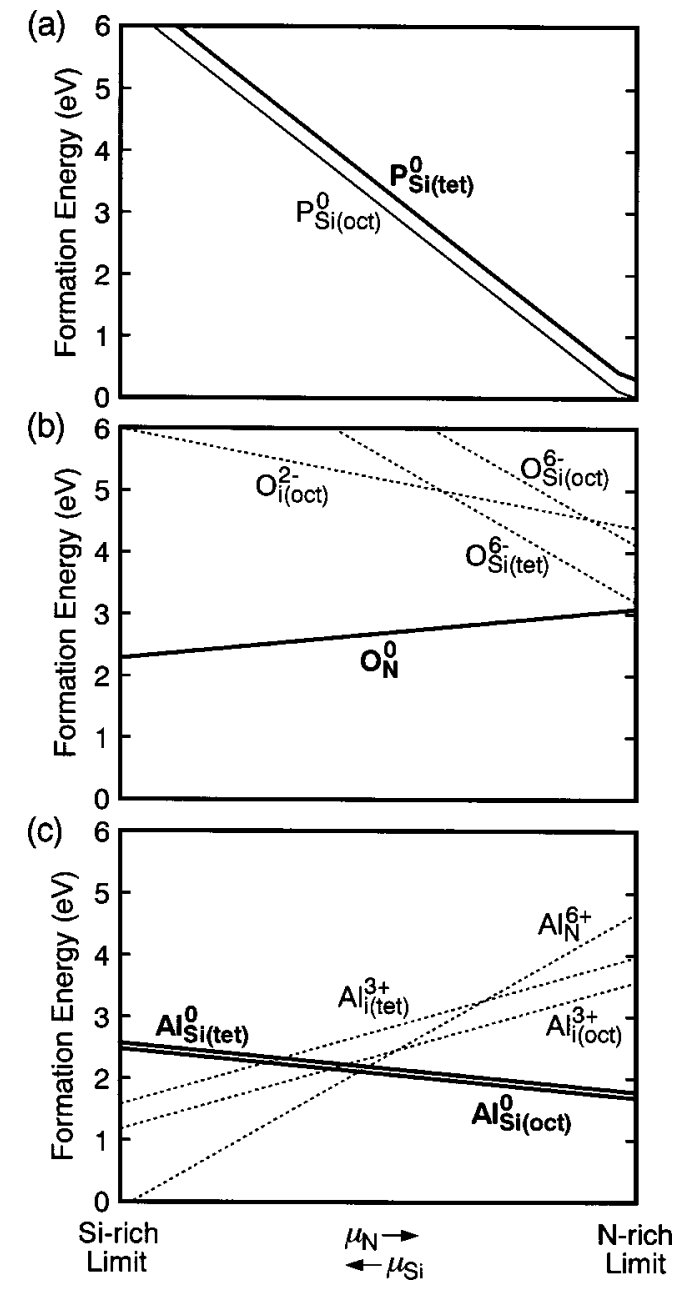

FIG. 1. Formation energies at the solubility limits as a function of the Si or $\mathrm{N}$ chemical potential for (a) $\mathrm{P}$, (b) $\mathrm{O}$, and (c) Al impurities. The Fermi energy is taken to be the minimum of the conduction band for $\mathrm{P}$ and $\mathrm{O}$ as $n$-type dopants, and to be the maximum of the valence band for $\mathrm{Al}$ as a p-type dopant. The superscripts indicate the charge states. The subscripts indicate the impurity sites; e.g., Si(tet) and i(oct) are the tetrahedral Si lattice site and the octahedral interstitial site of the $\mathrm{N}$ sublattice. For each site, the charge state that gives the lowest formation energy is shown. The formation energies of the impurities at the normal sites and the sites associated with compensation are shown with bold and dotted lines, respectively. 
We therefore propose that the $\mathrm{Al}$ impurities are the best candidate for $p$-type dopants.

We now consider the incorporation of the $\mathrm{P}, \mathrm{O}$, and $\mathrm{Al}$ impurities into other sites; these candidates may compensate carrier electrons or holes when they occupy anti or interstitial sites. Therefore, the calculation for these sites should be important. Figure 1 shows the formation energies of the P, O, and $\mathrm{Al}$ impurities occupying the normal substitutional sites that we discussed above, the other substitutional (anti) sites, and the tetrahedral and octahedral interstitial sites of the $\mathrm{N}$ sublattice. The formation energies are calculated at the solubility limits, assuming the Fermi energy to be the minimum of the conduction band for $\mathrm{P}$ and $\mathrm{O}$ as $n$-type dopants, and to be the maximum of the valence band for $\mathrm{Al}$ as a $p$-type dopant. For each impurity site, the charge state that gives the lowest formation energy is shown. The positive and negative charge states at anti and interstitial sites indicate that the impurities occupying these sites can compensate carrier holes and electrons, respectively.

Concerning the normal substitutional sites, N-rich conditions are preferable in formation energy for the $\mathrm{P}$ and $\mathrm{Al}$ impurities, while Si-rich conditions are favorable for the $\mathrm{O}$ impurities as mentioned above. Under these conditions, the formation energies of the $\mathrm{P}, \mathrm{O}$, and $\mathrm{Al}$ impurities at the antistitial and interstitial sites are quite higher than those for the normal sites. This implies that the compensation of carriers should not be a serious problem. However, the formation energies are dependent on the atomic chemical potentials, as recognized in Fig. 1. When we choose the opposite conditions of the chemical potentials, the formation energies for the normal sites and the sites associated with compensation become comparable or overturned for the $\mathrm{O}$ and $\mathrm{Al}$ impurities. The choice of growth conditions is therefore very important for effective doping.

In summary, we have investigated the formation and ionization energies of the impurities in $c-\mathrm{Si}_{3} \mathrm{~N}_{4}$ through first- principle calculations. The $\mathrm{P}$ and $\mathrm{O}$ impurities are suggested to be preferable for the $n$-type doping, while the $\mathrm{Al}$ impurities are favorable for the $p$-type doping. The compensation of doped carriers associated with the incorporation of these impurities into anti and interstitial sites can be suppressed if appropriate growth conditions are chosen. The present information on the dopants should be useful in the search of semiconducting $c-\mathrm{Si}_{3} \mathrm{~N}_{4}$.

The supports by the Grant-in-Aid for Scientific Research on Priority Areas (No. 751) from Ministry of Education, Science, Sports and Culture of Japan, and the ACT program from Japan Science and Technology Corporation are acknowledged.

${ }^{1}$ A. Zerr, G. Miehe, G. Serghiou, M. Schwarz, E. Kroke, R. Riedel, H. Fueß, P. Kroll and R. Boehler, Nature (London) 400, 340 (1999).

${ }^{2}$ S.-D. Mo, L. Ouyang, W. Y. Ching, I. Tanaka, Y. Koyama and R. Riedel, Phys. Rev. Lett. 83, 5046 (1999); The calculated band gap may be somewhat understimated owing to the local density approximation. The experimental band gap has not been reported.

${ }^{3}$ Y.-N. Xu and W. Y. Ching, Phys. Rev. B 51, 17379 (1995).

${ }^{4}$ V. Milman, B. Winkler, J. A. White, C. J. Pickard, M. C. Payne, E. V. Akhmatskaya, and R. H. Nobes, Int. J. Quantum Chem. 77, 895 (2000); The present calculations were performed using the CASTEP program code. (Molecular Simulations, Inc., San Diego, CA).

${ }^{5}$ J. P. Perdew, J. A. Chevary, S. H. Vosko, K. A. Jackson, M. R. Pederson, D. J. Singh, and C. Fiolhais, Phys. Rev. B 46, 6671 (1992).

${ }^{6}$ D. Vanderbilt, Phys. Rev. B 41, 7892 (1990).

${ }^{7}$ Calculated to be $98.0 \%$ of an experimental one in Ref. 1 .

${ }^{8}$ S. B. Zhang and J. E. Northrup, Phys. Rev. Lett. 67, 2339 (1991).

${ }^{9}$ C. G. Van de Walle, D. B. Laks, G. F. Neumark, and S. T. Pantelides, Phys. Rev. B 47, 9425 (1993).

${ }^{10}$ Calculated to be $3.6 \mathrm{eV}$ above the maximum of the valence band. This energy was evaluated as $\left\{E^{(1)}-E^{(0)}\right\}-\left\{E^{(0)}-E^{(-1)}\right\}$, where $E^{(N)}$ indicates the total energy of the perfect lattice supercell with additional $\mathrm{N}$ electrons. The value agrees with a band gap in the one-electron band structure.

${ }^{11}$ Under the equilibrium conditions, the chemical potentials of the impurities were determined using the total energies of the simple substances and the compounds, which were calculated after the optimization of the structure. 